\title{
Generation and collision of optical similaritons in dispersion-engineered silicon photonic nanowires
}

\author{
Spyros Lavdas ${ }^{a}$, Jeffrey B. Driscoll ${ }^{b}$, Richard R. Grote $^{b}$, Richard M. Osgood, Jr, ${ }^{b}$ and \\ Nicolae C. Panoiu ${ }^{a}$ \\ ${ }^{a}$ Department of Electronic and Electrical Engineering, University College of London, \\ Torrington Place WC1E 7JE, London, United Kingdom; \\ ${ }^{b}$ Microelectronics Sciences Laboratories, Columbia University, NY 10027, New York, USA
}

\begin{abstract}
We present a comprehensive study of generation and collision of optical similaritons in sub-micron silicon photonic wire waveguides. Our analysis of optical pulse dynamics in such wave guiding devices is based on a rigorous theoretical model that incorporates all of the relevant linear and nonlinear optical effects, including modal dispersion, free-carrier dispersion and absorption, self-phase modulation, two-photon absorption, frequency dispersion of the optical nonlinearity, and the free-carrier dynamics. In addition to the particular characteristics of the generation of optical similaritons in silicon photonic wires, we also investigate the dependence of the efficiency of this optical process on the physical parameters and temporal profile of the input pulse. The collision of optical similaritons that propagate both in the normal and anomalous dispersion regime is also analyzed. Guided by the target applications of our study, we considered two technologically relevant spectral regions, namely, telecom and mid-IR frequency domains.
\end{abstract}

Keywords: Optical similaritons, silicon waveguides, optical pulse propagation.

\section{INTRODUCTION}

The rapid increase of data transmission rate in many optical communication systems has led to an intense interest in generating pulses that do not suffer from distortion and wave breaking phenomena upon propagation. An example of such type of pulses that preserve their shape during propagation and show particle-like behavior are localized optical waves also known as solitons. They exist in the anomalous group-velocity dispersion (GVD) regime, namely when the second-order dispersion coefficient, $\beta_{2}<0$. Similar types of pulses that show particlelike properties and self-similar behavior upon propagation are the so-called similaritons. ${ }^{1,2,3,4,5}$ Unlike solitons, they form primarily in the normal dispersion regime $\left(\beta_{2}>0\right)$, although the existence of similaritons in the anomalous GVD regime has been demonstrated as well. ${ }^{6}$ Similaritons preserve their overall pulse intensity and phase profiles, although specific pulse parameters, such as the pulse width, amplitude, and frequency chirp vary during propagation. As a result, similaritons are not affected by destructive effects, such as pulse distortion, radiative damping, and wave breaking phenomena. More importantly, from a practical point of view, in order to generate optical similaritions no specific constraints have to be imposed on the physical parameters describing the initial pulse. The generation of optical similaritons and their physical properties have already been studied and demonstrated in optical fiber systems such as Yb-doped fiber amplifiers, ${ }^{2}$ using passive schemes based on dispersion managed or tapered silica fibers, ${ }^{4}$ and high-power amplifiers. ${ }^{7}$

The constant drive towards increased photonic systems integration makes it necessary to develop innovative approaches to generate at chip-scale optical pulses that are not distorted when propagated in subwavelength wave guiding photonic devices. Recent studies ${ }^{8}$ in which it is demonstrated that similaritons can form in silicon (Si) fibers with micrometer sized core represent an important step towards this goal. However, device fabrication considerations suggest that the silicon-on-insulator (SOI) material platform ${ }^{9,10}$ provides a more flexible and cost-effective solution to further decrease the device footprint. By scaling down the transverse device size to submicrometer range, the enhanced optical field confinement not only leads to larger modal frequency dispersion but also increases considerably the effective optical nonlinearity of the waveguide. As such, significant pulse reshaping effects can be achieved over relatively short propagation distance. For example, soliton generation in Si photonic nanowires (SiPhNWs) has already been demonstrated experimentally in chip-scale devices. ${ }^{11}$ Other important

\footnotetext{
Nanoengineering: Fabrication, Properties, Optics, and Devices X, edited by Eva M. Campo,

Elizabeth A. Dobisz, Louay A. Eldada, Proc. of SPIE Vol. 8816, 88160J · C 2013 SPIE

CCC code: $0277-786 X / 13 / \$ 18 \cdot$ doi: $10.1117 / 12.2023728$
}

Proc. of SPIE Vol. 8816 88160J-1 
nonlinear optical effects that can be used efficiently to engineer the intensity and phase profiles of optical pulses, such as supercontinuum generation, ${ }^{12,13,14}$ pulse self-steepening, ${ }^{15}$ and modulational instability ${ }^{16}$ have been demonstrated both at telecom $(\lambda=1.55 \mu \mathrm{m})$ and mid-IR $(\lambda \gtrsim 2.2 \mu \mathrm{m})$ wavelengths (see Refs. $[17,18,19]$ and references therein for more information on nonlinear optical effects in Si waveguides). The latter spectral domain provides an additional advantage for using $\mathrm{Si}$ as material platform for integrated nonlinear photonic devices, namely, the two-photon absorption (TPA) of Si is dramatically reduced.

In this article, we present a comprehensive analysis of formation and collision of similaritons in SiPhNWs. We consider similaritons that form in the normal and anomalous GVD regimes at both telecom and mid-IR spectral domains. Our article is organized as follows: In the next section we introduce a rigorous theoretical model on which our analysis of pulse dynamics is based. Then, in Sec. 3 we briefly outline the wavelength and waveguide width dependence of the main physical parameters that influence the pulse dynamics. Section 4 presents a detailed study of the physical characteristics of similariton generation in SiPhNWs whereas in Sec. 5 we discuss the collision between such optical pulses. Finally, in the last section the main conclusions are summarized.

\section{THEORETICAL MODEL FOR OPTICAL PULSE PROPAGATION}

Our study is based on a widely used rigorous theoretical mode ${ }^{20}$ that incorporates all of the relevant linear and nonlinear optical effects that influence the pulse dynamics upon propagation in SiPhNWs, including modal frequency dispersion, free-carrier (FC) dispersion and FC absorption, self-phase modulation (SPM), TPA, frequency dispersion of the optical nonlinearity, and the FC dynamics. The frequency dispersion and waveguide width dependence of the physical parameters of our model are rigorously accounted for, which allows us to apply our model to pulse propagation in width-modulated $\mathrm{SiPhNWs}^{21,22}$ and at both telecom and mid-IR frequencies.

Thus, the pulse dynamics are described by the following partial differential equation: ${ }^{19,20,21,22}$

$$
i \frac{\partial u}{\partial z}+\sum_{n \geq 1} \frac{i^{n} \beta_{n}(z)}{n !} \frac{\partial^{n} u}{\partial t^{n}}=-\frac{i c \kappa(z)}{2 n v_{g}(z)} \alpha_{\mathrm{FC}}(z) u-\frac{\omega \kappa(z)}{n v_{g}(z)} \delta n_{\mathrm{FC}}(z) u-\gamma(z)\left[1+i \tau(z) \frac{\partial}{\partial t}\right]|u|^{2} u,
$$

where $u(z, t)$ is the pulse envelope, normalized to units of $\sqrt{\mathrm{W}}, z$ and $t$ are the distance along the SiPhNWs and time, respectively, $\beta_{n}(z)=d^{n} \beta / d \omega^{n}$ is the $n$th order dispersion coefficient, $\kappa(z)$ is a physical parameter that quantifies the overlap between the optical mode and the active area ( $\mathrm{Si}$ core) of the waveguide, $v_{g}(z)$ is the group-velocity, $\delta n_{\mathrm{FC}}(z)=-\left(e^{2} / 2 \epsilon_{0} n \omega^{2}\right)\left[N(z) / m_{c e}^{*}+N(z)^{0.8} / m_{c h}^{*}\right]$ is the FC induced index change, $\alpha_{\mathrm{FC}}(z)=\left(e^{3} N(z) / \epsilon_{0} c n \omega^{2}\right)\left(1 / \mu_{e} m_{c e}^{* 2}+1 / \mu_{h} m_{c h}^{*}{ }^{2}\right)$ is the FC induced loss coefficient, where $N$ is the FC density, $m_{c e}^{*}=0.26 m_{0}$ and $m_{c h}^{*}=0.39 m_{0}$ are the effective mass of the electrons and holes, respectively, with $m_{0}$ the mass of the electron, and $\mu_{e}$ and $\mu_{h}$ the electron and hole mobility, respectively.

The nonlinear properties of the waveguide are chiefly described by the effective nonlinear waveguide coefficient, $\gamma(z)=3 \omega \Gamma(z) / 4 \epsilon_{0} A(z) v_{g}^{2}(z)$, where $A(z)$ and $\Gamma(z)$ are the cross-sectional area and the effective third-order susceptibility of the waveguide, respectively. When the pulse duration approaches the shock time scale, i.e. the characteristic response time of the waveguide nonlinearity, $\tau(z)=\partial \ln \gamma(z) / \partial \omega$, the frequency dispersion of the waveguide nonlinearity must be considered as well. Our theoretical model is completed by a standard rate equation describing the dynamics of the FCs,

$$
\frac{\partial N}{\partial t}=-\frac{N}{t_{c}}+\frac{3 \Gamma^{\prime \prime}(z)}{4 \epsilon_{0} \hbar A^{2}(z) v_{g}^{2}(z)}|u|^{4},
$$

where $\Gamma^{\prime \prime}$ and $\Gamma^{\prime}$ are the imaginary and real part of $\Gamma$, respectively, and $t_{c}$ is the recombination time.

\section{DISPERSION PROPERTIES OF SILICON PHOTONIC WIRE WAVEGUIDES}

The SiPhNW considered in this study consists of a Si rectangular core with an adiabatically varying width, $w(z)$, which is buried in $\mathrm{SiO}_{2}$. The height of the waveguide is assumed to be constant, $h=250 \mathrm{~nm}$. In order to incorporate in our model the $z$-dependence of the optical parameters of the waveguide we determine the fundamental (TE-like) optical mode and its propagation constant for 51 values of $w$, ranging from $500 \mathrm{~nm}$ to 

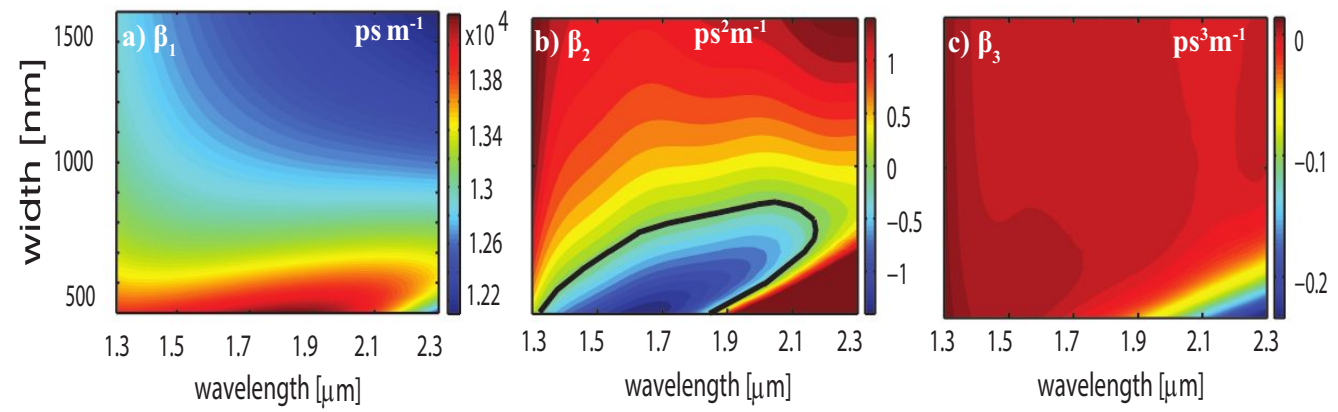

Figure 1. Width dependence of the spectra of the waveguide dispersion coefficients: a) $\beta_{1}$, b) $\beta_{2}$, and c) $\beta_{3}$. The black solid curve in b) corresponds to $\beta_{2}=0$.

$1500 \mathrm{~nm}$. In each case, the spectral domain in which these physical quantities are calculated is $1.3 \mu \mathrm{m}<\lambda<$ $2.3 \mu \mathrm{m}$. The optical modes are then used to determine the physical parameters $\kappa(\lambda), \gamma(\lambda)$, and $\tau(\lambda)$ for all values of $w$. Finally, by fitting the dependence $\beta(\lambda)$ with a 12th-order polynomial, for all values of $w$, and taking the corresponding derivatives we determine the higher-order dispersion coefficients. In the cases in which $w$ does not belong to the set of the 51 calculated values we use polynomial interpolation to calculate the corresponding values of the waveguide parameters.

The width dependence of the spectra of the waveguide dispersion coefficients are presented in Fig. 1. These plots show that the linear waveguide parameters depend strongly on both $w$ and $\lambda$, which provides increased flexibility in choosing the regime in which the device operates. In particular, it can be seen that if $w<887 \mathrm{~nm}$ the $\mathrm{SiPhNW}$ has two zero GVD wavelengths, defined by the relation $\beta_{2}(\lambda, w)=0$. In contrast, when $w>887 \mathrm{~nm}$ the $\mathrm{SiPhNW}$ has normal GVD in the entire spectral domain. However, if $\lambda>2187 \mathrm{~nm}$ the waveguide has normal GVD for any width. Moreover, within the range of the waveguide parameters investigated here, $\beta_{2}$ decreases monotonically with $w$ if $\lambda<1.78 \mu \mathrm{m}$, whereas if $\lambda>1.78 \mu \mathrm{m}$ the dependence $\beta_{2}(w)$ has a minimum point. A strong dependence on the waveguide width is also shown by $\beta_{1}=1 / v_{g}$, namely, $\beta_{1}$ decreases as $w$ increases.

Pulse propagation in SiPhNWs is not only determined by the waveguide dispersion coefficients but also by the nonlinear coefficients, which are plotted in Fig. 2. Specifically, the magnitude of the real and imaginary parts of the waveguide nonlinearity coefficient, $\gamma$, which describe the SPM and TPA effects, respectively, increase as $w$ and $\lambda$ decrease. As a result, it is expected that pulse reshaping phenomena induced by optical nonlinear effects will be more intense at lower wavelengths in SiPhNWs with smaller $w$. On the other hand, the shock time parameter shows a different dependence on the waveguide width. Specifically, whereas $\tau^{\prime}$ increases as $w$ decreases, $\tau^{\prime \prime}$ varies only slightly with $w$. Note that $\tau^{\prime}$ and $\tau^{\prime \prime}$ are time scales associated to different nonlinear effects, namely, SPM and TPA, respectively.
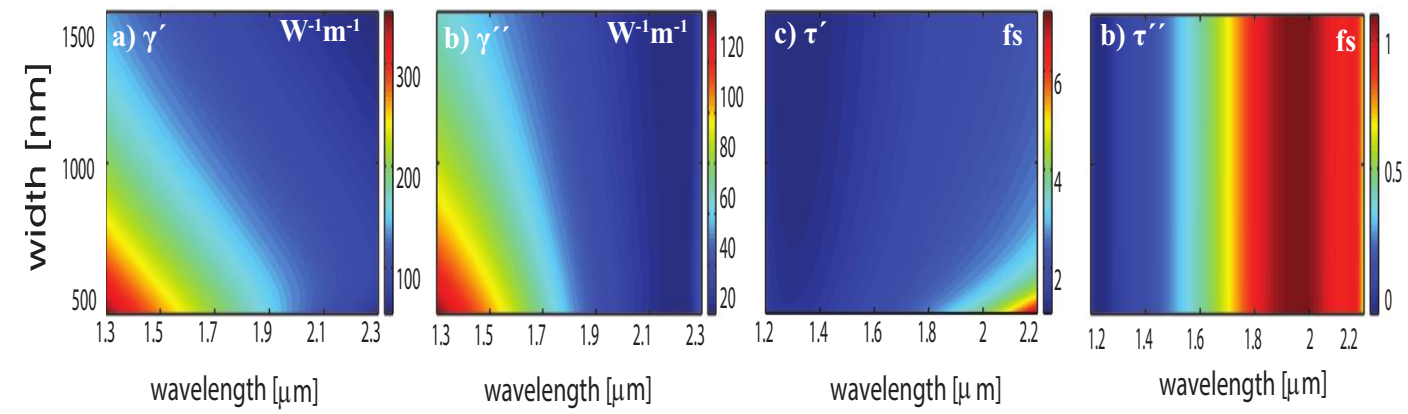

Figure 2. Width dependence of the spectra of the nonlinear waveguide coefficients: a) SPM parameter, $\gamma^{\prime}$, b) TPA coefficient, $\gamma^{\prime \prime}, \mathrm{c}$ ) shock time coefficient (real part), $\tau^{\prime}$, and d) shock time coefficient (imaginary part), $\tau^{\prime \prime}$. 


\section{GENERATION OF OPTICAL SIMILARITONS}

For optical similaritons to be used in photonics related applications there must exist easily available, versatile techniques for their generation. To this end, in this section we demonstrate that indeed optical similaritons can be easily generated from pulses whose parameters are practically free from physical constraints. To illustrate this, we consider first Gaussian pulses, $u_{\text {in }}(t)=e^{-\frac{1}{2}\left(\frac{t}{T_{0}}\right)^{2}}$, with full-width at half-maximum (FWHM), $T_{\mathrm{FWHM}}=200 \mathrm{fs}$ $\left(T_{\mathrm{FWHM}}=1.665 T_{0}\right)$, and peak power $P_{0}=10 \mathrm{~W}$, which are launched into an exponentially tapered SiPhNW. The taper function that describes the dependence of the width on the propagation distance is the following: $w(z)=w_{\text {in }}+\left(w_{\text {out }}-w_{\text {in }}\right)\left(1-e^{-a z}\right) /\left(1-e^{-a L}\right)$, with $w_{\text {in }}=1500 \mathrm{~nm}, w_{\text {out }}=820 \mathrm{~nm}, a=0.2 \mathrm{~m}^{-1}$ describes the rate at which the width of the taper changes, and $L$ is the taper length. We investigate the efficiency of the similariton formation process in two different cases, namely at telecom and mid-IR wavelengths. Under these circumstances, the waveguide parameters are: (i) at $\lambda=1.55 \mu \mathrm{m}, \beta_{2, \text { in }}=1.11 \mathrm{ps}^{2} \mathrm{~m}^{-1}, \beta_{2 \text {,out }}=0.31 \mathrm{ps}^{2} \mathrm{~m}^{-1}$, and $L=3 \mathrm{~mm}$; and (ii) at $\lambda=2.2 \mu \mathrm{m}, \beta_{2, \text { in }}=1.54 \mathrm{ps}^{2} \mathrm{~m}^{-1}, \beta_{2, \text { out }}=0.046 \mathrm{ps}^{2} \mathrm{~m}^{-1}$, and $L=6 \mathrm{~mm}$.

The evolution of the pulse profile and that of the density of photo-generated FCs, determined at both wavelengths, is depicted in Fig. 3. One effect illustrated by the pulse propagation presented in this figure is that, as expected, a stronger pulse decay is observed at $\lambda=1.55 \mu \mathrm{m}$. This is due to decreased TPA at mid-IR wavelengths [see also Fig. 2(b)]. Another interesting effect is revealed by the evolution of the density of FCs, namely, while at $\lambda=1.55 \mu \mathrm{m} N$ decreases with $z$, at $\lambda=2.2 \mu \mathrm{m}$, surprisingly, $N$ increases with $z$. To understand this behavior of the FC dynamics one can use Eq. (2) to infer that when the pulse width $T_{0} \ll t_{c}$, a condition which is satisfied in our simulations, the amount of generated FCs is $\Delta N(z) \sim P^{2}(z) \Gamma^{\prime \prime}(z) T_{\mathrm{FWHM}}(z) / A^{2}(z) v_{g}^{2}(z)$. The parameters $\Gamma^{\prime \prime}, T_{\mathrm{FWHM}}$, and $v_{g}$ vary only slightly with $z$, so that the main contribution to $\Delta N(z)$ comes from the $z$-variation of the ratio $P^{2}(z) / A^{2}(z)$. At $\lambda=1.55 \mu \mathrm{m}$ the pulse decays at a higher rate than the rate at which the taper narrows, so that $N$ decreases with $z$. By contrast, at $\lambda=2.2 \mu \mathrm{m}$ the pulse is absorbed at a much smaller rate, so that the dominant contribution to $\Delta N(z)$ primarily comes from the narrowing of the taper, meaning that $N$ increases with $z$. We point out that variations of $N$ induce changes in the effective index of the waveguide, and as such our results suggest that pump pulses can be used to induce a controllable optical environment that would be probed by a co-propagating optical signal.

An important phenomenon illustrated by the pulse dynamics shown in Fig. 3, which is the most relevant one for the topics investigated in this work, is the evolution of the initial Gaussian pulses into a parabolic one. The intensity profile of parabolic pulses is described by $\left|u_{p}(t)\right|^{2}=\left|u_{p}\left(t_{0}\right)\right|^{2}\left[1-\left(t-t_{0}\right)^{2} / T_{p}^{2}\right]$ for $\left|t-t_{0}\right|<T_{p}$

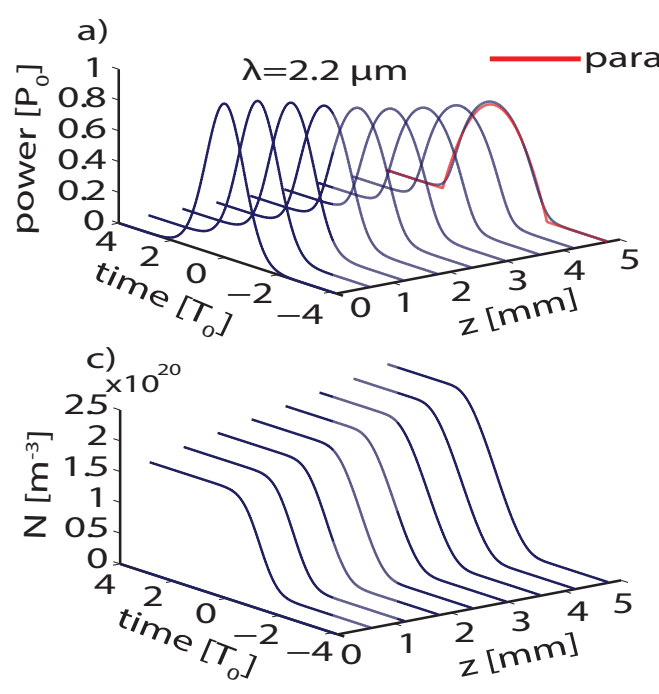

b)

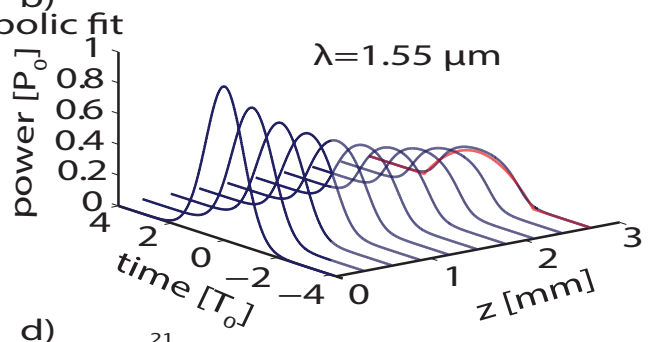

d)

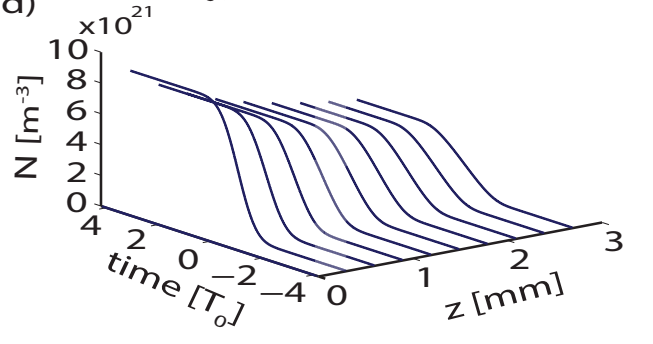

Figure 3. Evolution of pulse profile (top panels) and FC-density (bottom panels) in a tapered SiPhNW. The numerical simulations are performed at $\lambda=2.2 \mu \mathrm{m}$ (left panels) and $\lambda=1.55 \mu \mathrm{m}$ (right panels). The red curves correspond to the fitted parabolic pulses. 
and $u_{p}(t)=0$ otherwise, where $u_{p}\left(t_{0}\right), t_{0}$, and $T_{p}$ are the amplitude, time shift, and pulse width, respectively, whereas the phase of such pulses depends quadratically on time. It should be noted that in terms of the required propagation distance for the formation of parabolic similaritons, this optical process is more effective at $\lambda=1.55 \mu \mathrm{m}$. This is primarily due to the fact that the nonlinear waveguide coefficient, $\gamma^{\prime}$, is larger at telecom wavelengths [see Fig. 2(a)], which means that the nonlinear optical effects that are responsible for the pulse reshaping are enhanced as well.

In order to quantify the generation of parabolic pulses we use the intensity misfit parameter, $\varepsilon_{I}$, which provides a global measure of the degree to which the intensity profile of a pulse matches that of a parabolic one:

$$
\varepsilon_{I}^{2}=\frac{\int_{-\infty}^{\infty}\left[|u(t)|^{2}-\left|u_{p}(t)\right|^{2}\right]^{2} d t}{\int_{-\infty}^{\infty}|u(t)|^{4} d t} .
$$

For the case presented in Fig. 3, the minimum values of the misfit parameter are $\epsilon_{I}^{2}=1.4 \times 10^{-3}$ at $\lambda=2.2 \mu \mathrm{m}$, and is reached at $z=4.79 \mathrm{~mm}$, whereas $\epsilon_{I}^{2}=1.6 \times 10^{-3}$ for $\lambda=1.55 \mu \mathrm{m}$, and this minimum value is reached when $z=2.69 \mathrm{~mm}$. Our simulations also show that the frequency of the pulse becomes linearly dependent on time, across almost the entire temporal width of the pulse. The small values of $\epsilon_{I}^{2}$ and linearly chirped pulse frequency clearly demonstrate that parabolic similaritons are formed.

Parabolic similaritons can be generated from pulses other than Gaussian ones, the particular shape of the input pulse influencing only the similariton formation length. To demonstrate this, we considered input pulses with different shapes, namely, Gaussian, super-gaussian, $u(t)=e^{-\frac{1}{2}\left(\frac{t}{T_{0}}\right)^{2 m}}$ with $m=2\left(T_{\mathrm{FWHM}}=1.824 T_{0}\right)$, and hyperbolic-secant profile, $u(t)=\operatorname{sech}\left(t / T_{0}\right)$, in which case $T_{\mathrm{FWHM}}=1.763 T_{0}$. In all cases, the propagation distance $z=15 \mathrm{~mm}$ and $T_{\mathrm{FWHM}}=200 \mathrm{fs}$. Figure 4 summarizes the results of this analysis, namely, it shows the $z$-dependence of $\epsilon_{I}^{2}$ calculated for different input powers. This figure suggests that Gaussian pulses are the most suitable for generating parabolic pulses, since for this pulse profile the values of the misfit parameter are the smallest. In addition, for Gaussian and sech pulses the band of small values of the misfit parameter is wider at $\lambda=2.2 \mu \mathrm{m}$ than it is at $\lambda=1.55 \mu \mathrm{m}$. In both cases the width of these bands becomes narrower as $P_{0}$ increases. On the other hand, super-gaussian pulses at $\lambda=2.2 \mu \mathrm{m}$ are characterized by two bands of small misfit parameter values, whereas in the case of $\lambda=1.55 \mu \mathrm{m}$ there is only one such band, which broadens as $P_{0}$ increases. Figure 4 also reveals that of all pulse shapes considered super-gaussian pulses are characterized by the shortest similariton formation length. This conclusion is expected since of all three shapes the profile of a super-gaussian pulse is closest to a parabolic one.

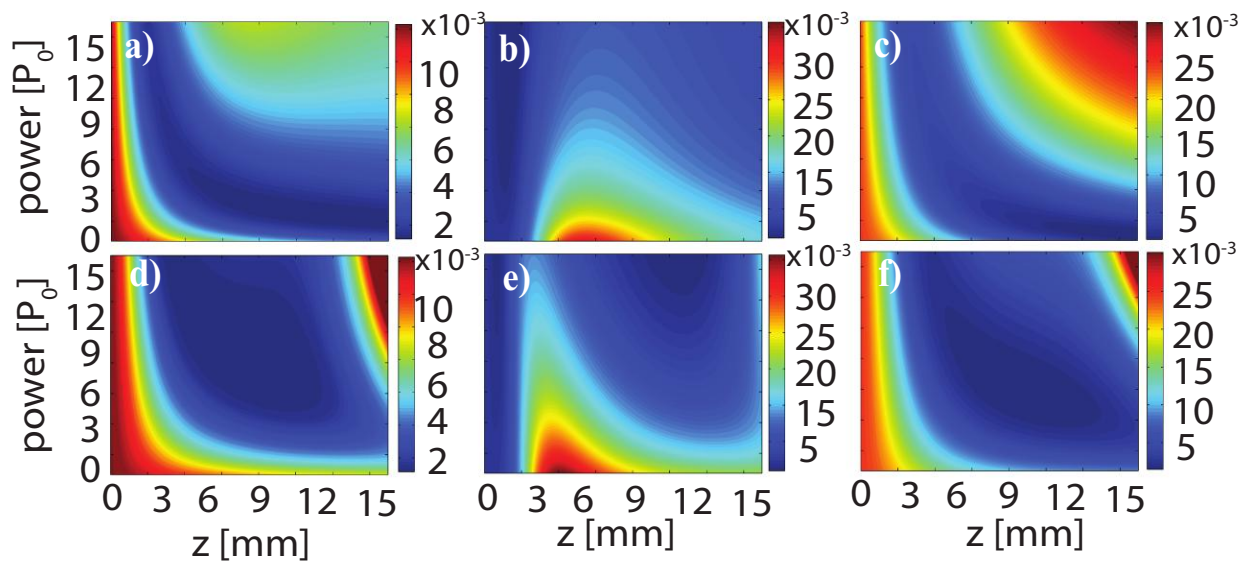

Figure 4. Dependence of $\epsilon_{I}^{2}$ on propagation distance and peak power, calculated for different pulse shapes: a) and d) Gaussian, b) and e) super-gaussian, and c) and f) sech pulse. Top and bottom panels correspond to $\lambda=1.55 \mu \mathrm{m}$ and $\lambda=2.2 \mu \mathrm{m}$, respectively. 


\section{COLLISION OF OPTICAL SIMILARITONS}

The results of our study presented so far demonstrate that parabolic similaritons can be easily generated in tapered SiPhNWs. However, one important physical property of such pulses, which is particularly relevant to technological applications, is that similaritons display particle-like behavior during collision. It should be noted that one expects that the knowledge pertaining to similaritons collision in regular or photonic crystal fibers ${ }^{23,24}$ cannot be simply translated to the context of SiPhNWs, as the basic optical properties of these optical guiding systems make them quite distinct from each other. For example, the presence of FCs in SiPhNWs means that optical similaritons collide in these waveguides in the presence of nonlinear losses induced by the TPA, whereas in the case of silica based optical fibers optical losses depend linearly on the pulse amplitude.

In order to study the collision of optical similaritons in SiPhNWs, we launch in these waveguides two such pulses with different carrier frequencies, namely, $\Omega_{0}+\Delta \Omega$ and $\Omega_{0}-\Delta \Omega$. This amounts to the two pulses having different group-velocities. The pulses are temporally separated by $\Delta t$, which can be arbitrarily chosen. We consider first the case of normal GVD, so that, for simplicity, we assume that the width of the waveguide is constant, $w=900 \mathrm{~nm}$. The initial profile of the optical field is then given by:

$$
u_{p}(t)=A_{01} \sqrt{1-\frac{(t-\bar{t})^{2}}{T_{0}^{2}}} e^{i[\phi(t-\bar{t})+\Delta \Omega(t-\bar{t})]}+A_{02} \sqrt{1-\frac{(t+\bar{t})^{2}}{T_{0}^{2}}} e^{i[\phi(t+\bar{t})-\Delta \Omega(t+\bar{t})]},
$$

where $A_{01}$ and $A_{02}$ are the amplitudes of the two similaritons and $\bar{t}=\Delta t / 2$. Moreover, the parameters of the similaritons are related by the following relations: ${ }^{3}$

$$
\begin{aligned}
\phi(t) & =\phi_{0}-\frac{3 \gamma^{\prime}}{4 \gamma^{\prime \prime}}+\frac{\gamma^{\prime \prime} A_{0}^{2}}{3 \beta_{2}} t^{2}, \\
A_{0} & =\frac{\gamma^{\prime \prime} E_{\text {in }}}{2 \sqrt{2 \gamma^{\prime} \beta_{2}}}, \\
T_{0} & =\frac{6 \gamma^{\prime} \beta_{2}}{{\gamma^{\prime \prime}}^{2} E_{\text {in }}},
\end{aligned}
$$

where $\phi_{0}$ is a constant phase and $E_{\text {in }}$ is the energy of the input pulse. In deriving these relations we assumed that the initial (nonlinear) loss coefficient is $\alpha_{\mathrm{nl}}=-2 \gamma^{\prime \prime} A_{0}^{2}$. To determine the initial values of the parameters of the colliding similaritons we choose first the pulse width, $T_{0}$, assumed to be the same for the two similaritons, then using Eq. (5c) we calculate the initial pulse energy, $E_{\text {in }}$, and from Eq. (5b) we find the amplitude of the similaritons, $A_{0}$. Finally, the initial phase profile is determined from Eq. (5a). Since $\beta_{2}$ and $\gamma$ are frequency dependent, the two similaritons will be characterized by different sets of parameters.

We have applied this procedure to study the collision of two parabolic similaritons with $\lambda_{0}=1.55 \mu \mathrm{m}$ and frequency shift $\Delta \Omega=130.2 \mathrm{THz}$, which means that the wavelengths of the two similaritons are $\lambda_{1}=1400 \mathrm{~nm}$ and
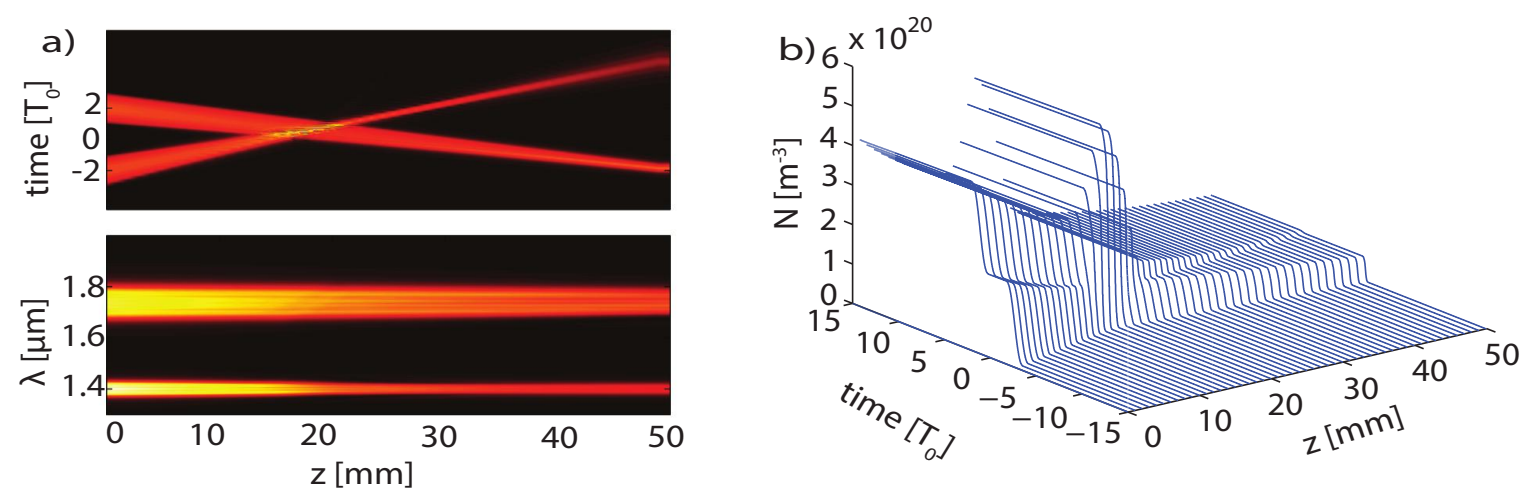

Figure 5. Collision of two parabolic similaritons propagating in the normal GVD regime and in the presence of large TPA. 

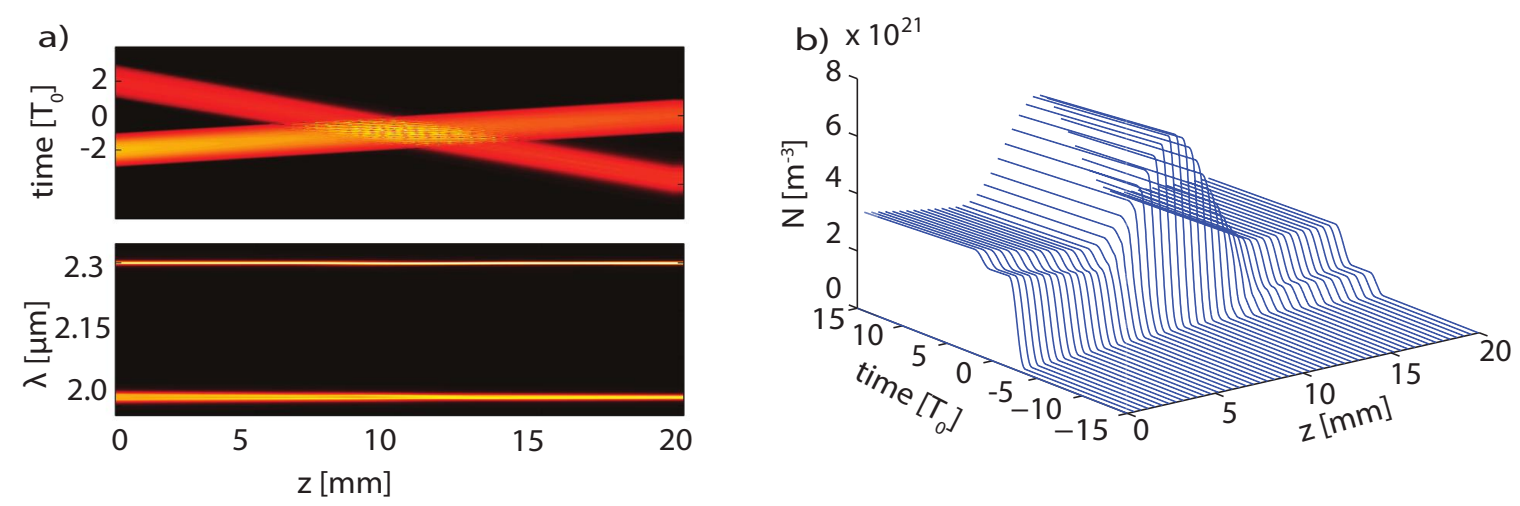

Figure 6. Collision of two parabolic similaritons propagating in the normal GVD regime and in the presence of weak TPA.

$\lambda_{2}=1736 \mathrm{~nm}$. The pulse width was set to $T_{0}=600 \mathrm{fs}$, the remaining pulse parameters being $P_{01}=440.4 \mathrm{~mW}$ and $P_{02}=447.8 \mathrm{~W}$. This choice of the parameters of the input pulses ensures that the wavelengths of both similaritons lie in a region of large TPA. The results of our simulations, presented in Fig. 5, clearly illustrate the particle-like behavior of the similaritons upon the pulse collision. Specifically, it can be seen that, except of some slight interference effects observed in the overlap region, the similaritons pass through each other being practically unaffected by the collision process. In addition, the strong TPA leads to significant pulse absorption, which is also reflected in the dependence of the FC-density on the propagation distance. In particular, one can observe a significant decrease with $z$ of the FC density, except, as expected, in the overlap region where a larger amount of FCs is generated. In addition to the collision of the similaritons, Fig. 5 also illustrates their temporal compression, which is their expected dynamics in a medium with optical losses.

We also considered the collision of parabolic similaritons propagating at mid-IR wavelengths. Thus, we chose $\lambda_{0}=2.2 \mu \mathrm{m}$ and $\Delta \Omega=57 \mathrm{THz}$, the corresponding wavelengths of the two similaritons being $\lambda_{1}=2019 \mathrm{~nm}$ and $\lambda_{2}=2300 \mathrm{~nm}$. With a pulse width of $T_{0}=2.5 \mathrm{ps}$, the peak powers of the two similaritons were found to be $P_{01}=2.54 \mathrm{~W}$ and $P_{02}=1.62 \mathrm{~W}$. Since the spectral separation between the two similaritons is smaller in this case, we have chosen a waveguide width at which the group-velocity is more dispersive at $\lambda_{0} \sim 2.2 \mu \mathrm{m}$, namely, $w=530 \mathrm{~nm}$. Figure 6 illustrates the dynamics of the similaritons collision in this case. While the evolution of the optical field again shows that the similaritons are not destroyed as a result of their collision, the dynamics of FCs are more complex as compared to what we have observed in the previous case. Specifically, it can be seen that an increased amount of FCs is generated while the two similaritons collide, whereas the FC-density remains rather constant when the two pulses are temporally separated. Interestingly enough, the similariton at $\lambda_{1}=2019 \mathrm{~nm}$ decays faster than the one at $\lambda_{2}=2300 \mathrm{~nm}$, although it probes a lower FC-density and thus smaller FC absorption. This result, explained by the reduced TPA experienced by the latter similariton, demonstrates that in this spectral regime and for pulse parameters considered here the TPA is significantly larger than the FC absorption. Moreover, the reduced TPA at mid-IR wavelengths also results in a smaller pulse compression.

In order to complete our analysis of similaritons interaction in SiPhNWs we considered the collision of similaritons that propagate in the anomalous GVD regime. Since in the mid-IR the waveguide has normal GVD for any width, in this case we only study the similaritons collision at $\lambda_{0}=1.55 \mu \mathrm{m}$. Moreover, for the two pulses to propagate in the anomalous GVD regime we set $w=510 \mathrm{~nm}$. In the case of anomalous GVD, the similariton profile is described by a hyperbolic-secant function, so that the input pulse is written as: ${ }^{6}$

$$
u_{p}(t)=\frac{1}{T_{0}} \sqrt{\frac{\left|\beta_{2}\left(\lambda_{1}\right)\right|}{\gamma^{\prime}\left(\lambda_{1}\right)}} \operatorname{sech}\left(\frac{t-\bar{t}}{T_{0}}\right) e^{i[\phi(t-\bar{t})+\Delta \Omega(t-\bar{t})]}+\frac{1}{T_{0}} \sqrt{\frac{\left|\beta_{2}\left(\lambda_{2}\right)\right|}{\gamma^{\prime}\left(\lambda_{2}\right)}} \operatorname{sech}\left(\frac{t+\bar{t}}{T_{0}}\right) e^{i[\phi(t+\bar{t})-\Delta \Omega(t+\bar{t})]} .
$$

Here, $\phi(t)=\phi_{0}-\left(\gamma^{\prime \prime} P_{0} / \beta_{2}\right) t^{2}, \phi_{0}$ being a constant phase. Note that in the case when the quadratic chirp term in this relation vanishes the corresponding sech pulses are the well-known solitons. They correspond to a case of a SiPhNW with constant optical parameters and no optical losses or gain. As it is well known, solitons would 

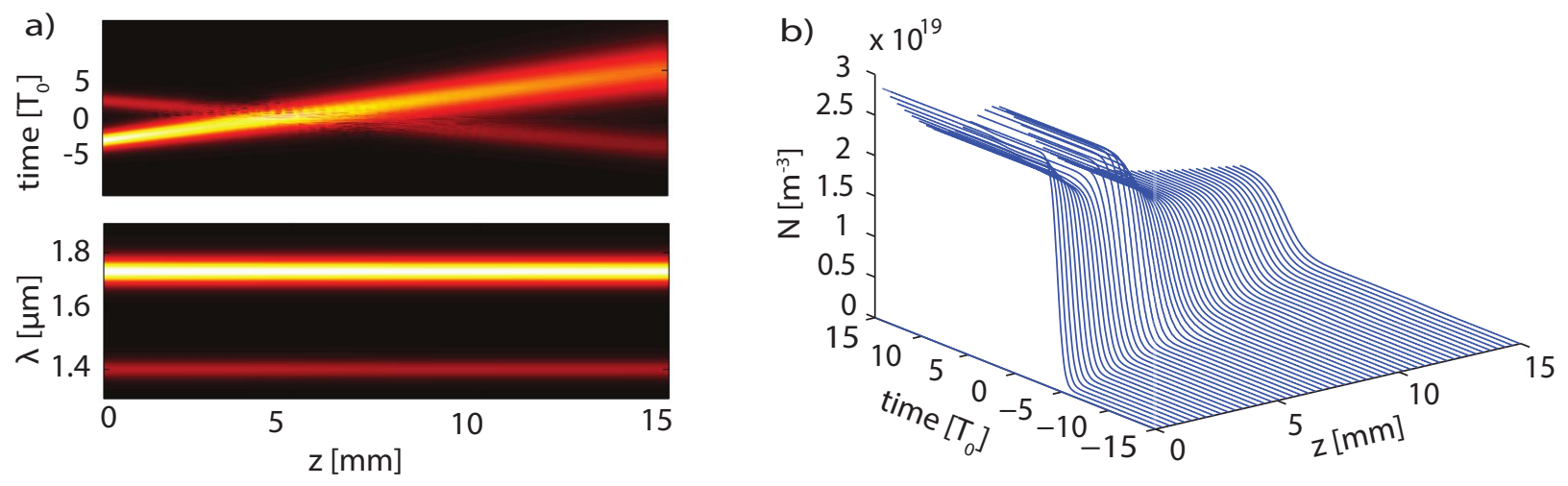

Figure 7. Collision of two parabolic similaritons propagating in the anomalous GVD regime and in the presence of large TPA.

propagate in such waveguides without any change in their shape or pulse parameters, whereas similaritons only preserve their shape upon propagation.

As in the case of normal GVD, we set the frequency shift $\Delta \Omega=130.2 \mathrm{THz}$, meaning that $\lambda_{1}=1400 \mathrm{~nm}$ and $\lambda_{2}=1736 \mathrm{~nm}$. With a pulse width $T_{0}=200 \mathrm{fs}$, the pulse peak powers are $P_{01}=48 \mathrm{~mW}$ and $P_{02}=154 \mathrm{~mW}$. The collision of sech similaritons, presented in Fig. 7, has several features that distinguish this case from that of parabolic similaritons. First, it can be seen that although both similaritons are compressed during the propagation, an effect similar to that of soliton compression, in this case the compression factor is much smaller. Second, Fig. 7 shows that the dynamics of FCs is chiefly determined by the propagation of just one of the two similaritons. This result is easily explained by the quadratic dependence of the generated FCs on the pulse power, combined with the fact that the optical power of one of the similaritons is more than three times larger than the peak power of the other one. Despite these differences, however, in this case too the sech similaritons show particle-like behavior during collision.

\section{CONCLUSIONS}

In conclusion, we have demonstrated that parabolic similaritons can be easily generated in tapered Si-PhNWs. Our analysis showed that using this approach optical similaritons can be generated at both telecom and mid-IR wavelengths, from pulses that are readily available in standard experimental set-ups. In particular, we considered Gaussian, super-gaussian, and hyperbolic-secant pulses, our study revealing that the smallest similariton formation length corresponds to super-gaussian pulses. Finally, we also considered the collision of optical similaritons propagating in the normal and anomalous GVD regimes, in both cases the dynamics of the optical field and those of photo-generated FCs being investigated. This analysis has demonstrated that although the optical similaritons are not destroyed in the collision process, the similaritons interaction is strongly dependent on the particular conditions in which the collision occurs, including the frequency dispersion regime, spectral domain, and pulse parameters.

\section{ACKNOWLEDGMENTS}

The work of S. L. was supported through a UCL Impact Award graduate studentship. R. R. G. acknowledges support from the Columbia Optics and Quantum Electronics IGERT under NSF Grant DGE-1069420.

\section{REFERENCES}

[1] Anderson, D., Desaix, M., Karlsson, M., Lisak M., and Quiroga-Teheiro, M. L., "Wave breaking free pulses in nonlinear optical fibers," J. Opt. Soc. Am. B 10, 1185-1190 (1993).

[2] Kruglov, V. I., Peacock, A. C., Harvey, J. D., and Dudley, J. M., "Self-similar propagation of parabolic pulses in normal-dispersion fiber amplifiers," J. Opt. Soc. Am. B 19, 461-469 (2002). 
[3] Fermann, M. E., Kruglov, V. I., Thomsen, B. C., Dudley, J. M., and Harvey, J. D., "Self-similar propagation and amplification of parabolic pulses in optical fibers," Phys. Rev. Lett. 84, 6010-6013 (2000).

[4] Hirooka, T., and Nakazawa, M., "Parabolic pulse generation by use of a dispersion-decreasing fiber with normal-group velocity dispersion," Opt. Lett. 29, 498-500 (2004).

[5] Ponomarenko, S. A., and Agrawal, G. P., "Optical similaritons in nonlinear waveguides," Opt. Lett. 32, 1659-1661 (2007).

[6] Kruglov, V. I., Peacock, A. C., and Harvey, J. D., "Exact self-similar solutions of the generalized nonlinear Schrodinger equation with distributed coefficients," Phys. Rev. Lett. 90, 113902 (2003).

[7] Limpert, J., Schreiber, T., Clausnitzer, T., Zollner, K., Fuchs, H. J., Kley, E. B., Zellmer, H., and Tunnermann, A., "High-power femtosecond Yb-doped fiber amplifier," Opt. Express 10, 628-638 (2002).

[8] Peacock, A., and Healy, N., "Parabolic pulse generation in tapered silicon fibers," Opt. Lett. 35, 1780-1782 (2010).

[9] Lee, K. K., Lim, D. R., Luan, H. C., Agarwal, A., Foresi, J., and Kimerling, L. C., "Effect of size and roughness on light transmission in a $\mathrm{Si} / \mathrm{SiO}_{2}$ waveguide: Experiments and model," Appl. Phys. Lett. 77, 1617-1619 (2000).

[10] R. U. Ahmad, F. Pizzuto, G. S. Camarda, R. L. Espinola, H. Rao, and R. M. Osgood, IEEE Photon. Technol. Lett. 14, 65-67 (2002).

[11] Hsieh, I. W., Chen, X., Dadap, J. I., Panoiu, N. C., Osgood, R. M., McNab, S. J., and Vlasov, Y. A., "Ultrafast-pulse self-phase-modulation and third-order dispersion in Si photonic wire-waveguides," Opt. Express 14, 12380-12387 (2006).

[12] Hsieh, I. W., Chen, X., Liu, X. P., Dadap, J. I., Panoiu, N. C., Chou, C. Y., Xia, F., Green, W. M., Vlasov, Y. A., and R. M. Osgood, "Supercontinuum generation in silicon photonic wires," Opt. Express 15, $15242-15249$ (2007).

[13] Boyraz, O., Koonath, P., Raghunathan, V., and Jalali, B., "All optical switching and continuum generation in silicon waveguides," Opt. Express 12, 4094-4102 (2004).

[14] Kuyken, B., Liu, X. P., Osgood, R. M., Baets, R., Roelkens, G., and Green, W. M. J., "Mid-infrared to telecomband supercontinuum generation in highly nonlinear silicon-on-insulator wire waveguides," Opt. Express 19, 20172-20181 (2011).

[15] Panoiu, N. C., Liu, X., and Osgood, R. M., "Self-steepening of ultra short pulses in silicon photonic nanowires," Opt. Lett. 34, 947-949 (2009).

[16] Panoiu, N. C., Chen, X., and Osgood, R. M., "Modulation instability in silicon photonic nanowires," Opt. Lett. 31, 3609-3611 (2006).

[17] Lin, Q., Painter, O. J., and Agrawal, G. P., "Nonlinear optical phenomena in silicon waveguides: Modeling and applications," Opt. Express 15, 16604-16644 (2007).

[18] Dadap, J. I., Panoiu, N. C., Chen, X. G., Hsieh, I. W., Liu, X. P., Chou, C. Y., Dulkeith, E., McNab, S. J., Xia, F. N., Green, W. M. J., Sekaric, L., Vlasov, Y. A., and Osgood, R. M., "Nonlinear-optical phase modification in dispersion-engineered Si photonic wires," Opt. Express 16, 1280-1299 (2008).

[19] Osgood, R. M. , Panoiu, N. C., Dadap, J. I., Liu, X., Chen, X., Hsieh, I-W, Dulkeit, E., Green, W. M. J., and Vlassov, Y. A., "Engineering nonlinearities in nanoscale optical systems: physics and applications in dispersion engineered silicon nanophotonics wires," Adv. Opt. Photon. 1, 162-235 (2009).

[20] Chen, X., Panoiu, N. C., and Osgood, R. M., "Theory of Raman-mediated pulsed amplification in siliconwire waveguides," IEEE J. Quantum Electron. 42, 160-170 (2006).

[21] Panoiu, N. C., McMillan, J. F., and Wong, C. W., "Theoretical analysis of pulse dynamics in silicon photonic crystal wire waveguides," IEEE J. Sel. Top. Quantum Electron. 16, 257-266 (2010).

[22] Driscoll, J. B., Ophir, N., Grote, R. R., Dadap, J. I., Panoiu, N. C., Bergman, K., and Osgood, R. M., "Width-modulation of Si photonic wires for quasi-phase-matching of four-wave-mixing: experimental and theoretical demonstration," Opt. Express 20, 9227-9242 (2012).

[23] Finot, C., and Millot, G., "Collisions between similaritons in optical fiber amplifiers," Opt. Express 13, 7653-7663 (2005).

[24] Ponomarenko, S. A., and Agrawal, G. P., "Nonlinear interaction of two or more similaritons in loss- and dispersion-managed fibers," J. Opt. Soc. Am. B 25, 983-989 (2008). 